\title{
Recycling Cellular Downlink Energy for Overlay Self-Sustainable IoT Networks
}

\author{
Fatma Benkhelifa*, Hesham ElSawy**, Julie A. McCann*, and Mohamed-Slim Alouini*** \\ * Imperial College London, London, UK \\ ** King Fahd University of Petroleum and Minerals (KFUPM), Dhahran, Saudi Arabia \\ *** King Abdullah University of Science and Technology (KAUST), Thuwal, Makkah Province, Saudi Arabia \\ \{f.benkhelifa, j.mccann\}@imperial.ac.uk, \{elsawyhesham\}@gmail.com, \{slim.alouini\}@kaust.edu.sa
}

\begin{abstract}
This paper investigates the self-sustainability of an overlay Internet of Things (IoT) network that relies on harvesting energy from a downlink cellular network. Using stochastic geometry and queueing theory, we develop a spatiotemporal model to derive the steady state distribution of the number of packets in the buffers and energy levels in the batteries of IoT devices given that the IoT and cellular communications are allocated disjoint spectrum. Particularly, each IoT device is modeled via a two-dimensional discrete-time Markov Chain (DTMC) that jointly tracks the evolution of data buffer and energy battery. In this context, stochastic geometry is used to derive the energy generation at the batteries and the packet transmission probability from buffers taking into account the mutual interference from other active IoT devices. To this end, we show the Pareto-Frontiers of the sustainability region, which defines the network parameters that ensure stable network operation and finite packet delay. The results provide several insights to design self-sustainable IoT networks.

Index Terms-Spatiotemporal models, stochastic geometry, queuing theory, energy harvesting, packet transmission success probability, two-dimensional discrete-time Markov chain, stability conditions.
\end{abstract}

\section{INTRODUCTION}

The Internet of Things (IoT) is the paradigm that bridges the physical and cyber worlds such that everything and anything will be connected to the Internet. Such ubiquitous and massive connectivity has unlimited potentials to advance our life (e.g., smart cities, automated diagnostics, autonomous deriving, smart agriculture, public safety, etc.) [1]. Realizing the IoT paradigm brings potentially billions of new devices (e.g., sensors, actuators, machines, robots, vehicles, etc.) to the already congested wireless spectrum. In addition to the spectrum scarcity problem, the IoT intrinsic features impose several new challenges to conventional wireless networks. For instance, the IoT involves a multitude heterogeneity of devices with diverse quality of service requirements. Recharging/changing batteries of massive numbers of devices, that are distributed over a large scale area, represents another acute challenge to sustain the IoT operation. Hence, revolutionary wireless technologies are required to address the unprecedented challenges that arise in IoT networks [2], [3].

From the industrial perspective, several new technologies and standards are being developed to accommodate the potential surge of IoT traffic. For instance, the latest 3rd Generation Partnership Project (3GPP) amendments propose
Narrowband IoT (NB-IoT) and machine type communication (LTE-MTC) to accommodate IoT traffic within cellular networks [4]. Furthermore, several new low power wide area networks (LPWANs) are being developed and tailored for the IoT use cases such as Sigfox, Weightless and LoRa [3], [5]. The 3GPP amendments and the LPWAN account for the intrinsic features of the IoT such as massive density, sporadic traffic, multitude heterogeneity, and high-energy efficiency. At the device level, transceivers with energy harvesting capabilities are being developed [6], which circumvent the overwhelming administrative overhead of sustaining the IoT network.

In parallel to the industrial progress, the research community is developing rigorous mathematical paradigms to characterize, design, and operate IoT networks. In this context, stochastic geometry (see [7] for a tutorial) is indispensable when characterizing the performance of interference limited IoT networks [8]-[10]. However, stand-alone stochastic geometry fails to account for the sporadic traffic patterns of the IoT devices. Recently, several studies have integrated stochastic geometry and queueing theory to characterize the network performance while accounting for the spatiotemporal traffic generation. For instance, the work in [11] characterizes the delay and the maximum spatiotemporal traffic that uplink cellular networks can accommodate. The work in [12] studies the delay in downlink cellular networks with unsaturated traffic. The work in [13] studies the percentile based performance (e.g., transmissions success probability and delay) for a static ad hoc IoT network. However, the studies in [11]-[13] assume that all devices have perpetual energy sources. The problem of energy scarcity and harvesting for large-scale networks is considered in [14]-[17] via unsaturated energy queues. However, the studies in [14]-[17] assume saturated data buffers for all devices. Hence, none of the aforementioned works consider unsaturated data buffers and energy queues to study the self-sustainability of large scale IoT networks.

This paper proposes a novel spatiotemporal mathematical model, based on stochastic geometry and queueing theory, to characterize and design self-sustainable IoT networks. We consider an IoT network modeled via a Poisson bipolar process (PBP), where each device is equipped with a data buffer and an energy queue (i.e., battery). Data is generated, and stored for transmission, at each buffer according to 
an independent and identically distributed (i.i.d.) geometric distribution. Batteries are replenished by scavenging radio frequency (RF)-energy from a downlink cellular network, where the base-stations (BSs) are modeled via an independent Poisson point process (PPP). A transmission attempt occurs from an IoT device when the data buffer is nonempty and the harvested energy in the battery is sufficient for transmission. The transmission attempt is successful if the signal-to-interference ratio (SIR) at the receiver is above a certain threshold. Hence, each bipolar link is modeled via a two-dimensional discrete-time Markov Chain (DTMC) and the IoT network is considered as a network of spatially interacting DTMCs. To this end, we obtain the packet transmission success probability at the network steady state and characterize the self-sustainability region of the network. ${ }^{1}$ The main contributions in this paper are summarized below:

- To the best knowledge of the authors, this paper presents the first mathematical model that jointly accounts for the spatiotemporal traffic generation, the energy harvesting problem, and mutual interference between devices in a large scale IoT network.

- We show the Pareto-frontiers of the sustainability region, which characterize the maximum spatiotemporal traffic that an IoT network can accommodate via recycling the RF-power of a cellular network with a given BS density.

- We illustrate the spectrum scarcity and energy scarcity tradeoff within self-sustainable IoT networks.

\section{System Model}

For the sake of organized treatise, we discuss the spatial, temporal, and energy harvesting models in separate sections.

\section{A. Spatial and Propagation Models}

We consider an IoT network that is spatially distributed in $\mathbb{R}^{2}$ according to a homogeneous PBP with spatial intensity $\lambda_{D}$. Hence, the IoT network is constituted from IoT transmitter-receiver pairs, denoted as bipolar links, where each link has a fixed length of $r_{0}$ and a uniform random orientation $\varepsilon \in[0,2 \pi]$. According to the PBP, the IoT transmitting devices constitute a PPP, denoted as $\Phi_{D}=$ $\left\{y_{j}: j=1,2, \ldots\right\}$, where $y_{j} \in \mathbb{R}^{2}$ denotes the location of the $j$ 'th IoT transmitter. The IoT network coexists with a cellular network that is spatially distributed according to an independent PPP, denoted as $\Phi_{B}=\left\{z_{i}: i=1,2, \ldots\right\}$, with spatial intensity $\lambda_{B}$.

We consider a Rayleigh fading environment with i.i.d. unit variance channels. The channel gains between the BSs and the IoT devices are denoted by $h$ and the channel gains between IoT devices are denoted by $g$. All channels gains are assumed to be independent of the spatial locations. We utilize a power-law path loss model such that the signal power decays at the rate $r^{-\alpha}$, where $r$ is the propagation distance and $\alpha$ is the path loss exponent. The path loss exponent between IoT devices is denoted as $\alpha_{D}$, which is generally

\footnotetext{
${ }^{1}$ The self-sustainability region defines all network parameters that ensure stable data buffers and finite transmission delay across the network.
}

different from the path loss exponent between a BS and an IoT device, denoted as $\alpha_{B}$.

Each BS becomes active with probability $b$ independently of other BSs, where active BSs transmit with a fixed power $P_{B}{ }^{2}$ The IoT network operates at a dedicated spectrum that is universally reused by all the IoT devices. Hence, the IoT devices mutually interfere with each other but there is no interference between the cellular and IoT networks. Each IoT transmitter inverts its path-loss towards its associated receiver such that the useful average received power at each IoT receiver is maintained at $\rho_{D}$. Since the distance between the IoT transmitters and their intended IoT receivers is fixed, the required transmit power $P_{D}$ at each IoT transmitter is given by $P_{D}=\rho_{D} r_{0}^{\alpha_{D}}$.

\section{B. Temporal and Queuing Model}

We consider a discrete-time slotted system with slot duration of $T_{s}$ seconds. Each IoT transmitter is equipped with a data buffer. The data packets are generated at each IoT device according to an i.i.d. geometric distribution with parameter $a \in[0,1]$. For each device, the generated packets are stored in the buffer to be transmitted over the wireless channel to the intended receiver according to the first-in firstout (FIFO) discipline. Packets are transmitted one-by-one and a packet will be discarded from the buffer after being successfully transmitted to the intended receiver.

\section{Energy Harvesting Model}

Hence, all IoT transmitters rely on scavenging the downlink RF-energy from the cellular network to replenish their batteries. Hence, each IoT transmitter is equipped with an energy receiver and rechargeable battery (i.e., energy queue) for energy harvesting. The energy receiver is tuned to the downlink cellular frequency, which is different from the IoT dedicated frequency. As such, the IoT transmitters harvest only from the cellular network and are not influenced by the strong downlink interference.

The IoT transmitters employ the well-known "harvestthen-transmit" protocol. All IoT devices are equipped with a single antenna switching between the energy receiver and information transmitter. Hence, the IoT transmitter should keep harvesting and storing energy in its battery (i.e., energy queue) until the stored energy is sufficient to achieve the required power of $\rho_{D}$ at its associated receiver. Once the energy harvesting phase is completed, the IoT device switches to the transmission mode and transmit one packet from its data buffer if a packet exists. We denote the activity probability, i.e., the probability of having non-empty buffer and sufficient energy for transmission, with $d$. Without loss of generality, we assume a finite energy queue size that is sufficient for transmitting one packet. ${ }^{3}$ Once a transmission attempt occurs, the energy is depleted from the battery and the device returns to the harvesting phase.

\footnotetext{
${ }^{2}$ The activity factor $b$ reflects the time-varying user loads and traffic per BS.

${ }^{3}$ Such assumption enables us to study the interplay between the energy scarcity and spectrum scarcity problems in energy harvesting IoT networks.
} 


\section{Methodology of Analysis}

In many IoT use cases (e.g., smart parking meters), the IoT devices are fixed. Furthermore, in case of nomadic and low-mobility IoT scenarios, the fading and device/BS activity vary at much shorter time scale when compared to the time required to make tangible location displacement for IoT devices. Hence, it is reasonable to assume an arbitrary, but static, cellular and IoT networks realization where only fading and devices/BSs activity change over time. In such static network setting, each IoT device may have its own location dependent performance [13], [18], [19] in terms of harvesting and transmission success probability. However, the activity probabilities $b$ and $d$ mitigate such location dependent discrepancies (i.e., decrease the variance) across the IoT devices performance [11], [18]. Exploiting this fact, we use the following approximation:

Approximation 1: The spatially averaged harvesting probabilities and transmission success probability of a typical IoT device is representative to all IoT devices in the network.

Remark 1: The effect of Approximation 1 diminishes as $b$ and $d$ decrease as different realization of, respectively, active BSs from $\Phi_{B}$ and active devices from $\Phi_{D}$ appear in each time slot. Note that the foreseen ultra-densification of cellular networks will dramatically decrease the load served by each BS and will lead to small activity factor $b$. Furthermore, $d$ is already small in the depicted network model due to the sporadic pattern of the IoT traffic along with the employed "harvest-then-transmit" scheme.

Remark 2: The cellular network is not utilized for information transfer and is used for energy harvesting only. Hence, the aggregate downlink power at each time slot comes from the independently thinned PPP $\Phi_{B}$, with intensity $b \lambda_{B}$, and the nearest BS to the IoT device is not necessarily contributing to the harvested energy. Hence, the energy harvesting variance among the devices does not saturate when decreasing $b$ as in the case of success probability in downlink information transfer shown in [18].

Remark 3: Approximation 1 becomes exact in high mobility scenarios [19].

Remark 4: The analysis in this paper can be extended to the case of static network with high $b$ and/or $d$ by following the same methodology in [13], which is postponed to future extension.

For simplicity, we discretize the battery into $M$ energy levels of equal amount $\omega=P_{D} / M$. Let $\boldsymbol{p}=\left[p_{0}, p_{m}, \cdots, p_{M}\right]$, where $p_{m}, 0 \leq m \leq M$, is the spatially averaged probability that the harvested energy is sufficient for replenishing $m$ battery levels in one time slot. Furthermore, let $p_{c}$ denote the spatially averaged probability of successful packet transmission and let $(\cdot)=(1-\cdot)$ denote the probability complement operator. Exploiting Approximation 1, the microscopic (i.e., individual) behavior of any IoT device can be modeled via the two-dimensional DTMC shown in Fig. 1 to track the temporal evolution of the data (levels) and battery (phases). As shown in Fig. 1, the battery may recharge $m$ levels per time slot with probability $p_{m}$. On the other hand, a singlestep transition can occur between buffer states as only one

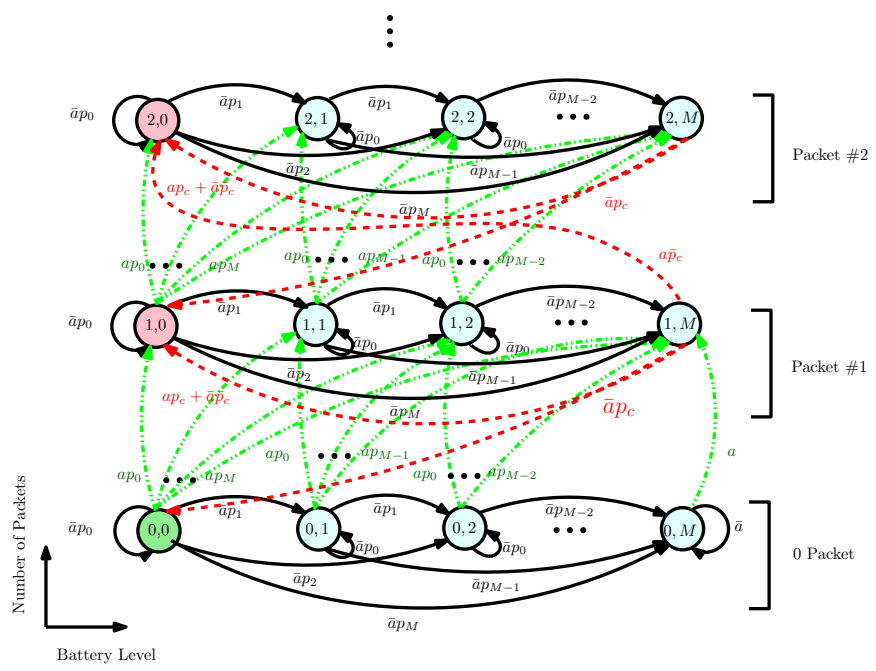

Figure 1. Two-dimensional discrete-time Markov chain modelling the evolution of the data buffer and the battery for each IoT device.

packet can be generated and/or transmitted per time slot. Note that a packet departure can only take place from the phase $M$ such that the stored energy is sufficient for a transmission attempt. After each transmission attempt, the battery is depleted and the DTMC goes to phase $m=0$ to reset the energy harvesting process. Note that while all IoT devices are represented via the same DTMC model, different IoT devices can have different buffer and battery states at a given time slot.

While the DTMC in Fig. 1 describes the microscopic behavior of an IoT device, the parameters $\boldsymbol{p}$ and $p_{c}$ are functions of the macroscopic (i.e., network wide) mutual interactions among all IoT devices and BSs. Particularly, the harvesting probability vector $\boldsymbol{p}$ is determined according to the distribution of the aggregate downlink power at the IoT transmitter from all active BSs. Furthermore, the transmission success probability $p_{c}$ is function of the interference power received by the IoT receiver from all other active IoT transmitters. Hence, stochastic geometry is employed to find the parameters $\boldsymbol{p}$ and $p_{c}$ in Section III-A and queueing theory is utilized to find the steady state distribution vector of the DTMC in Fig. 1 in Section III-B. Note that the stochastic geometry analysis and queueing theory analysis are interdependent because i) finding $p_{c}$ requires the intensity of mutually interfering IoT devices $d \lambda_{D}$; and ii) obtaining $d$ (i.e., from steady state probability of the DTMC) requires $p_{c}$. Such interdependence is solved via an iterative solutions in Section III-C based on the fixed point theorem.

\section{Spatiotemporal Model}

\section{A. Stochastic Geometry Analysis}

In this section, we use stochastic geometry to derive the spatially averaged transmission success probability $p_{c}$ and the energy harvesting probability vector $\boldsymbol{p}$. Without loss of generality, we focus on a test device located at an arbitrary origin, which becomes the typical device under spatial average. 
1) Packet Transmission: Let $\tilde{\Phi}_{D}$ be the point process of mutually interfering IoT devices and let the IoT receiver of the test bipolar IoT link be located at an arbitrary origin. A successful transmission occurs at the test link if the SIR at the test receiver is above a certain detection threshold $\theta$. Hence, the transmission success probability is given by

$$
p_{c}(\theta)=\mathbb{P}\left\{\frac{\rho_{D} g_{0}}{P_{D} \sum_{y_{j} \in \tilde{\Phi}_{D} \backslash y_{0}} g_{j}\left\|y_{j}\right\|^{-\alpha_{D}}}>\theta\right\},
$$

where $\|\cdot\|$ is the Euclidean norm. The transmission success probability is characterized via the following theorem.

Theorem 1: The packet transmission success probability for a typical IoT link is given by

$$
p_{c}(\theta)=\exp \left(-\frac{2 \pi^{2} d \lambda_{D} r_{0}^{2}}{\alpha_{D} \sin \left(\frac{2 \pi}{\alpha_{D}}\right)} \theta^{\frac{2}{\alpha_{D}}}\right) .
$$

Proof: The transmission success probability in (1) can be expressed, using the complementary density function (CDF) of exponential distribution with unit mean, as

$$
p_{c}(\theta)=\mathbb{E}_{y_{j}, g_{j}}\left[\exp \left(-\frac{\theta}{\rho_{D}} P_{D} \sum_{y_{j} \in \tilde{\Phi}_{D} \backslash y_{0}} g_{j}\left\|y_{j}\right\|^{-\alpha_{D}}\right)\right]=\mathcal{L}_{D}\left(\frac{\theta}{\rho_{D}}\right),
$$

where $\mathcal{L}_{D}(\cdot)$ is the Laplace transform (LT) of the aggregate interference derived in Appendix A in (22), which proves (2).

2) Energy Harvesting: For a given time slot, let $\tilde{\Phi}_{B}$ be the point process of simultaneously active BSs. Hence, the harvested power at a test IoT transmitter located at the origin is given by

$$
P_{H}=\zeta P_{B} \sum_{z_{i} \in \tilde{\Phi}_{B}} h_{i}\left\|z_{i}\right\|^{-\alpha_{B}},
$$

where $\zeta \in[0,1]$ is the harvesting efficiency. The harvested power $P_{H}$ is characterized in the following lemma:

Theorem 2: The CDF of the harvested power in a generic time slot is given by

$$
\begin{gathered}
F_{P_{H}}(x)=1-\frac{\alpha_{B}}{2 \pi} \int_{0}^{\infty} \frac{\exp \left(-x\left(\frac{\zeta^{\frac{2}{\alpha_{B}}} t}{\kappa_{0}}\right)^{\frac{\alpha_{B}}{2}}-t \cos \left(\frac{2 \pi}{\alpha_{B}}\right)\right) \sin \left(t \sin \left(\frac{2 \pi}{\alpha_{B}}\right)\right)}{t} d t \\
\stackrel{\left(\alpha_{B}=4\right)}{=} \operatorname{erfc}\left(\frac{\pi^{2} \delta}{4 \sqrt{x}}\right)
\end{gathered}
$$

where $\kappa_{0}=\frac{2 \pi^{2} b \lambda_{B} P_{B}^{\frac{2}{\alpha_{B}}}}{\alpha_{B} \sin \left(\frac{2 \pi}{\alpha_{B}}\right)}, \delta=b \lambda_{B} \sqrt{\zeta P_{B}}, \operatorname{erf}(z)=\frac{2}{\sqrt{\pi}} \int_{0}^{z} e^{-t^{2}} d t$ is the error function and $\operatorname{erfc}(\cdot)=1-\operatorname{erf}(\cdot)$ is the complementary error function.

Proof: See Appendix B.

Given that the battery is discretized into $M$ equal levels, the probability of harvesting $0 \leq m \leq M$ levels of energy is

$$
p_{m}= \begin{cases}F_{P_{H}}((m+1) \omega)-F_{P_{H}}(m \omega), & \text { if } m=0, \ldots, M-1, \\ 1-F_{P_{H}}(M \omega), & \text { if } m=M, \\ 0, & \text { otherwise. }\end{cases}
$$

For $\alpha_{B}=4$, we have the following corollary:

Corollary 1: For $\alpha_{B}=4$, the harvesting probabilities $p_{m}$ is given by

$p_{m} \underset{\alpha_{B}=4}{=} \begin{cases}\operatorname{erf}\left(\frac{\pi^{2} \delta}{4 \sqrt{m \omega}}\right)-\operatorname{erf}\left(\frac{\pi^{2} \delta}{4 \sqrt{(m+1) \omega}}\right), & \text { if } m=0, \ldots, M-1, \\ \operatorname{erf}\left(\frac{\pi^{2} \delta}{4 \sqrt{P_{D}}}\right), & \text { if } m=M .\end{cases}$

Proof: Follows from Theorem 2 and (7).

\section{B. Queueing Theory Analysis}

The DTMC in Fig. 1 represents a quasi birth death process (QBD) with geometric (Geo) arrival and phase $(\mathrm{PH})$ type departure, denoted as Geo/Ph/1 QBD system [20]. That is, the queue departure process (i.e., transmission success probability) can be represented via an absorbing Markov chain with the transient matrix

$$
\boldsymbol{S}=\left[\begin{array}{ccccc}
p_{0} & p_{1} & \ldots & p_{M-1} & 1-\sum_{m=0}^{M-1} p_{m} \\
0 & p_{0} & \ldots & p_{M-2} & 1-\sum_{m=0}^{M-2} p_{m} \\
\vdots & \ddots & \ddots & \vdots & \vdots \\
0 & \ldots & 0 & p_{0} & 1-p_{0} \\
\overline{p_{c}} & \ldots & \ldots & 0 & 0
\end{array}\right]
$$

The matrix $S$ is an $(M+1) \times(M+1)$ sub-stochastic matrix that models the harvesting process until one successful packet departure. Since a transmission attempt can only take place when the battery is full, then $\boldsymbol{s}=\boldsymbol{e}-\boldsymbol{S} \boldsymbol{e}=\left[0,0, \cdots, p_{c}\right]^{T}$, where $\boldsymbol{e}$ is a column vector of ones with the proper length. Since the batter is completely depleted with each transmission, the initialization vector for the $\mathrm{PH}$ type process is $\boldsymbol{\beta}=[1,0,0, \cdots, 0]$. When the IoT device has an empty buffer, it harvests energy until full battery and waits until a packet arrives. Hence, the energy harvesting matrix at level-0 is a stochastic matrix given by

$$
\boldsymbol{S}_{0}=\left[\begin{array}{ccccc}
p_{0} & p_{1} & \ldots & p_{M-1} & 1-\sum_{i=0}^{M-1} p_{i} \\
0 & p_{0} & \ldots & p_{M-2} & 1-\sum_{i=0}^{M-2} p_{i} \\
\vdots & \ddots & \ddots & \vdots & \vdots \\
0 & \ldots & 0 & p_{0} & 1-p_{0} \\
0 & \ldots & \ldots & 0 & 1
\end{array}\right] .
$$

Using the $\mathrm{PH}$ distributions described above, the transition matrix of the Geo/PH/1 system shown in Fig. 1 is given by

$$
\boldsymbol{P}=\left[\begin{array}{ccccc}
\boldsymbol{B} & \boldsymbol{C} & & & \\
\boldsymbol{A}_{2} & \boldsymbol{A}_{1} & \boldsymbol{A}_{0} & & \\
& \boldsymbol{A}_{2} & \boldsymbol{A}_{1} & \boldsymbol{A}_{0} & \\
& & \ddots & \ddots & \ddots
\end{array}\right]
$$

where $\boldsymbol{B}=\bar{a} \boldsymbol{S}_{0}$ and $\boldsymbol{C}=a \boldsymbol{S}_{0}$ are sub-stochastic matrices that contain the transition probabilities within the idle state and from the idle-to-level 1 (i.e., first packet arrival), respectively. The sub-stochastic matrices $\boldsymbol{A}_{0}=a \boldsymbol{S}, \boldsymbol{A}_{1}=a \boldsymbol{s} \boldsymbol{\beta}+\bar{a} \boldsymbol{S}$, and $\boldsymbol{A}_{2}=\bar{a} \boldsymbol{s} \boldsymbol{\beta}$ contain the buffer state transitions probabilities when going up one level, staying within the same level, and going down one level, respectively.

Let $\boldsymbol{x}=\left[\begin{array}{llll}\boldsymbol{x}_{0} & \boldsymbol{x}_{1} & \boldsymbol{x}_{2} & \ldots\end{array}\right]$, where $\boldsymbol{x}_{i}=\left[\begin{array}{lll}\boldsymbol{x}_{i, 0} & \ldots & x_{i, M}\end{array}\right]$ denotes the probability of having $i$ packets in the data buffer 
and $x_{i, m}$ is the probability of having $i$ packets and $m$ energy levels. The steady state vector $\boldsymbol{x}$ is obtained by solving

$$
\boldsymbol{x}=\boldsymbol{x} \boldsymbol{P} \quad \text { and } \quad \boldsymbol{x} \mathbf{1}=1
$$

where $\mathbf{1}$ is an infinite column vector of ones. A unique solution for the system of equations in (12) exists if the DTMC in Fig. 1 is stable. The stability condition is characterized as:

Lemma 1: A data buffer of an IoT device is stable if

$$
\pi A_{2} e>\pi A_{0} e
$$

where $\pi$ is defined as

$$
\boldsymbol{\pi}=\boldsymbol{e}^{T}\left(\boldsymbol{S}+\boldsymbol{s} \boldsymbol{\beta}-\boldsymbol{I}+\boldsymbol{e} \boldsymbol{e}^{T}\right)^{-1},
$$

and $\boldsymbol{I}$ is the identity matrix. Otherwise, the data buffer is unstable and the packet delay is infinite.

Proof: According to [20], a $\mathrm{Geo} / \mathrm{PH} / 1 \mathrm{QBD}$ system is stable if $\boldsymbol{\pi} \boldsymbol{A}_{2} \boldsymbol{e}>\boldsymbol{\pi} \boldsymbol{A}_{0} \boldsymbol{e}$, where $\boldsymbol{\pi}$ is solution to

$$
\boldsymbol{\pi}=\boldsymbol{\pi} \boldsymbol{A} \text { and } \boldsymbol{\pi} \boldsymbol{e}=1,
$$

where $\boldsymbol{A}=\boldsymbol{A}_{0}+\boldsymbol{A}_{1}+\boldsymbol{A}_{2}$. Substituting for $\boldsymbol{A}_{0}=a \boldsymbol{S}, \boldsymbol{A}_{1}=$ as $\boldsymbol{\beta}+\bar{a} \boldsymbol{S}$, and $\boldsymbol{A}_{2}=\bar{a} \boldsymbol{s} \boldsymbol{\beta}$ and using [21, Lemma 1] to solve (15), Lemma 1 is proved.

The subsequent analysis depends on the output of the stability condition in (13) as shown in the sequel.

1) Stable system: If the condition in (13) is satisfied, the solution for the DTMC in (12) is given as:

Theorem 3: The joint steady state probabilities for the number of data packet in the buffer and energy levels in the battery of a typical IoT device is given by

$\boldsymbol{x}_{i}= \begin{cases}a \boldsymbol{x}_{0} \boldsymbol{S}_{0}(\boldsymbol{I}-a \boldsymbol{s} \boldsymbol{\beta}-\bar{a} \boldsymbol{S}-\boldsymbol{R} \bar{a} \boldsymbol{s} \boldsymbol{\beta})^{-1} \bar{a} \boldsymbol{s} \boldsymbol{\beta}\left(\boldsymbol{I}-\bar{a} \boldsymbol{S}_{0}\right)^{-1}, & \text { if } i=0, \\ a \boldsymbol{x}_{0} \boldsymbol{S}_{0}(\boldsymbol{I}-a \boldsymbol{s} \boldsymbol{\beta}-\bar{a} \boldsymbol{S}-\boldsymbol{R} \bar{a} \boldsymbol{s} \boldsymbol{\beta})^{-1}, & \text { if } i=1, \\ \boldsymbol{x}_{1} \boldsymbol{R}^{i-1}, & \text { if } i>1\end{cases}$

satisfying $\boldsymbol{x}_{0} \boldsymbol{e}+a \boldsymbol{x}_{0} \boldsymbol{S}_{0}(\boldsymbol{I}-a \boldsymbol{s} \boldsymbol{\beta}-\bar{a} \boldsymbol{S}-\boldsymbol{R} \bar{a} \boldsymbol{s} \boldsymbol{\beta})^{-1}(\boldsymbol{I}-\boldsymbol{R})^{-1} \boldsymbol{e}=$ 1 , with $\boldsymbol{R}=a \boldsymbol{S}(\boldsymbol{I}-a \boldsymbol{s} \boldsymbol{\beta}-\bar{a} \boldsymbol{S}-a \boldsymbol{S e} \boldsymbol{\beta})^{-1}$.

Proof: Following [20], $\boldsymbol{x}_{0}$ and $\boldsymbol{x}_{1}$ are the solutions to:

$$
\left(\begin{array}{ll}
x_{0} & x_{1}
\end{array}\right)=\left(\begin{array}{ll}
x_{0} & x_{1}
\end{array}\right)\left(\begin{array}{cc}
B & C \\
A_{2} & A_{1}+R A_{2}
\end{array}\right) .
$$

where $\boldsymbol{R}$ is the minimal non-negative solution of the matrix quadratic equation $\boldsymbol{R}=\boldsymbol{A}_{0}+\boldsymbol{R} \boldsymbol{A}_{1}+\boldsymbol{R}^{2} \boldsymbol{A}_{2}$. Since $\boldsymbol{A}_{2}$ is ranked one [20], an explicit expression of $\boldsymbol{R}$ exists as

$$
\boldsymbol{R}=\boldsymbol{A}_{0}\left(\boldsymbol{I}-\boldsymbol{A}_{1}-\boldsymbol{A}_{0} \boldsymbol{e} \boldsymbol{\beta}\right)^{-1}=a \boldsymbol{S}(\boldsymbol{I}-a \boldsymbol{s} \boldsymbol{\beta}-\bar{a} \boldsymbol{S}-a \boldsymbol{S e} \boldsymbol{\beta})^{-1} .
$$

Note that if the system is stable in [20], $\boldsymbol{R}$ has a spectral radius less than one and the following results are obtained using this fact. Solving (17), $\boldsymbol{x}_{0}$ is solution to:

$$
\boldsymbol{x}_{0}=a \boldsymbol{x}_{0} \boldsymbol{S}_{0}(\boldsymbol{I}-a \boldsymbol{s} \boldsymbol{\beta}-\bar{a} \boldsymbol{S}-\boldsymbol{R} \bar{a} \boldsymbol{s} \boldsymbol{\beta})^{-1} \bar{a} \boldsymbol{s} \boldsymbol{\beta}\left(\boldsymbol{I}-\bar{a} \boldsymbol{S}_{0}\right)^{-1}
$$

with the normalisation condition boldsymbolx $x_{0} \boldsymbol{e}+$ $a_{0} \boldsymbol{x}_{0}(\boldsymbol{I}-a \boldsymbol{s} \boldsymbol{\beta}-\bar{a} \boldsymbol{S}-\boldsymbol{R} \bar{a} \boldsymbol{s} \boldsymbol{\beta})^{-1}(\boldsymbol{I}-\boldsymbol{R})^{-1} \boldsymbol{e}=1$. Solving for $\boldsymbol{x}_{0}, \boldsymbol{x}_{1}$ is deduced from (17) as in (16). For $i \geq 2, \boldsymbol{x}_{i}$ is given by $\boldsymbol{x}_{i}=\boldsymbol{x}_{1} \boldsymbol{R}^{i-1}$.
Let $\boldsymbol{\Phi}_{p}=\left[\Phi_{p, 0}, \Phi_{p, 1}, \ldots, \Phi_{p, M}\right]$ where $\Phi_{p, m}$ is the marginal probability of having $m$ units of energy in the battery for a non-empty data buffer. If the system is stable, the battery level marginal distribution is characterized in the following corollary:

Corollary 2: The marginal distribution of the phases with nonempty data buffers is given by

$$
\boldsymbol{\Phi}_{p}=\sum_{i \geq 1} \boldsymbol{x}_{i}=\boldsymbol{x}_{1}(\boldsymbol{I}-\boldsymbol{R})^{-1}
$$

Proof: Let $\boldsymbol{\Phi}=\left[\Phi_{0}, \Phi_{1}, \ldots, \Phi_{M}\right]$ where $\Phi_{m}$ be the marginal probability of having $m$ units of energy in the battery. The marginal distribution of the phases is given by $\boldsymbol{\Phi}=\sum_{i \geq 0} \boldsymbol{x}_{i}=\boldsymbol{x}_{0}+x_{1} \sum_{i \geq 1} \boldsymbol{R}^{i-1}=\boldsymbol{x}_{0}+\boldsymbol{x}_{1}(\boldsymbol{I}-\boldsymbol{R})^{-1}$. Since $\boldsymbol{\Phi}=\boldsymbol{x}_{0}+\boldsymbol{\Phi}_{p}$, we deduce (20).

Since an IoT device transmits when it has non-empty buffer and full battery, hence, the activity probability $d$ is

$$
d=\boldsymbol{\Phi}_{p, M} .
$$

2) Unstable system: If the condition in (13) is not satisfied, then the DTMC is unstable and Theorem 1 and Corollary 2 are no more applicable. This is because the departure rate is less than the arrival rate [20], and hence, the buffer accumulates infinite number of packets and the probability of having empty buffer is nullified. Although unstable buffers are never empty, an IoT device does not transmit unless it has full battery. Hence, only the marginal phase distribution for the battery is considered. This fact translates into setting $\boldsymbol{x}_{0}=0$, which leads to $\boldsymbol{\Phi}_{p}=\boldsymbol{e}^{T}\left(\boldsymbol{S}+\boldsymbol{s} \boldsymbol{\beta}-\boldsymbol{I}+\boldsymbol{e} \boldsymbol{e}^{T}\right)^{-1}$. Then, $d$ for unstable system is $d=\boldsymbol{\Phi}_{p, M}$.

\section{Iterative Solution}

As mentioned earlier, there is an interdependence between the microscopic device behavior and the macroscopic mutual interaction between IoT devices. Particularly, the aggregate interference seen by the typical IoT device is function of the activity of other IoT devices. Hence, the transmission success probability in (2) requires the probability that an IoT device is active, given by (21). Meanwhile, the queueing theory analysis for computing the activity probability in (21) requires the transmission success probability as shown in (9). Such interdependence is solved via the iterative solution in Algorithm 1, which converges to a unique solution by virtue of fixed point theorem [22, Appendix B].

\section{Numerical Results}

In this section, we first validate the proposed spatiotemporal mathematical framework against Monte Carlo simulations. Then, we discuss the self-sustainability of the depicted IoT network. Unless otherwise state, the network parameters are selected as follows: $P_{B}=1 \mathrm{~W}, \rho_{D}=-20$ $\mathrm{dBm}, r_{0}=2 \mathrm{~m}, \alpha_{B}=4, \alpha_{D}=3, \zeta=0.6$, and $M=8$.

The simulations are performed over a network area of $100 \times 100 \mathrm{~m}^{2}$, with a wrapped around boundaries. Two independent realizations of a PPP and a PBP, with intensities $\lambda_{B}=10^{-3} \mathrm{BSs} / \mathrm{m}^{2}$ and $\lambda_{D}=510^{-2} \mathrm{IoTs} / \mathrm{m}^{2}$, are used respectively. The realizations of the PPP and PBP are kept 

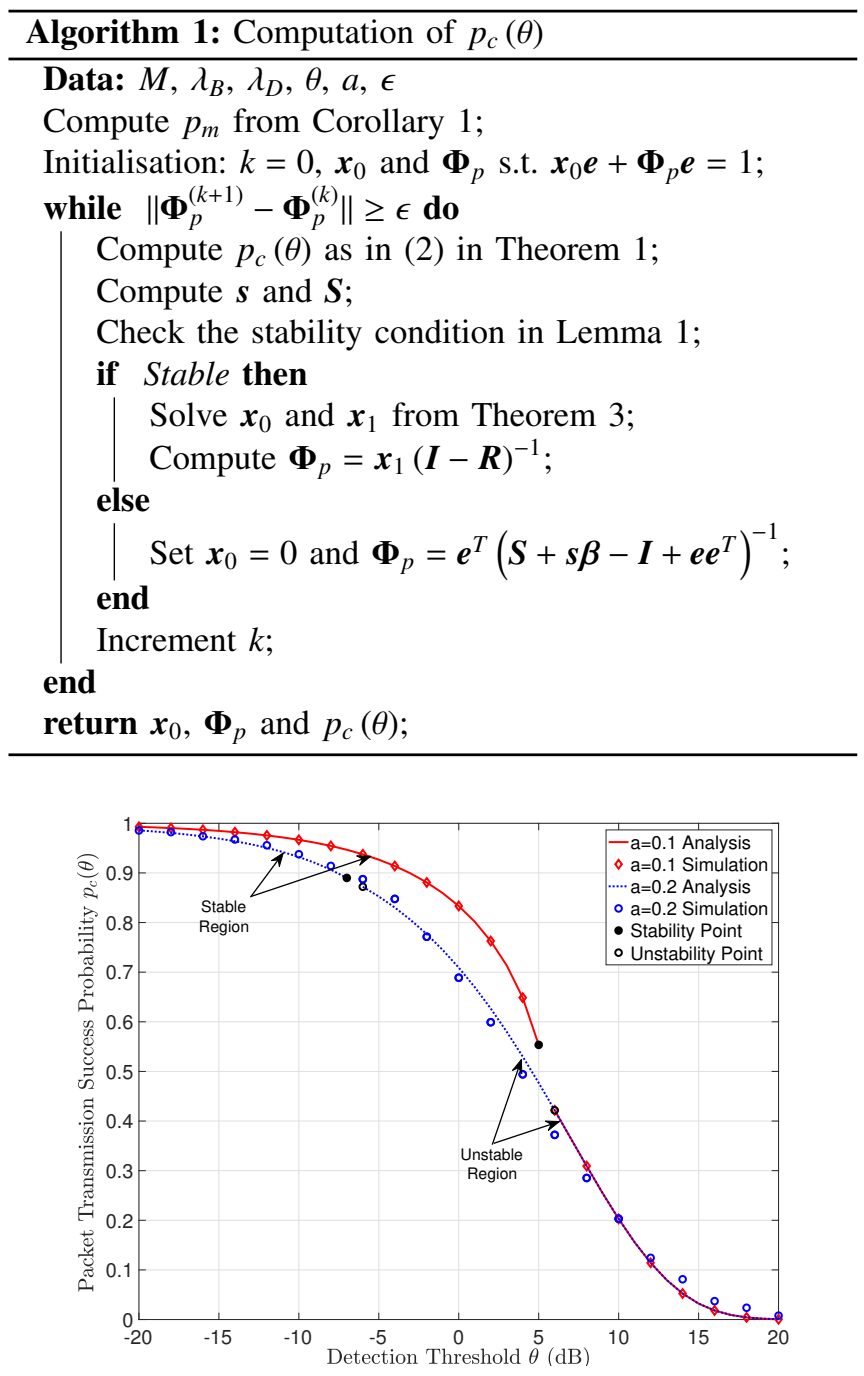

Figure 2. Packet transmission success probability $p_{c}(\theta)$ versus the detection threshold $\theta$ in $\mathrm{dB}$ for $a=0.1$ and $a=0.2$

fixed, while fading and devices/BSs activities change over time. Each simulation run is considered as a time slot where i.i.d. channel gains are instantiated, packets are generated, energy is harvested by some IoT devices and packets are transmitted by other IoT devices (i.e., those with non-empty buffers and full batteries). The time evolves and the transmission success probabilities and steady state statistics are traced until steady state, where the statistics are collected. Such process is repeated several times with different realizations of the PPP and PBP for the sake of spatial averaging.

Fig. 2 shows the analytic (i.e., Algorithm 1) and simulations for the packet transmission success probability $p_{c}(\theta)$ versus the detection threshold $\theta$ in $\mathrm{dB}$ for $a=0.1$ and $a=0.2$. First, the agreement between the analytical results and the Monte Carlo simulations validates the analysis. The figure shows that as the detection threshold $\theta$ increases, the packet transmission success probability decreases. Hence, the system becomes unstable when the transmission success probability leads to a departure rate that is less than the arrival rate. For $a=0.2$ and $a=0.1$ the system becomes

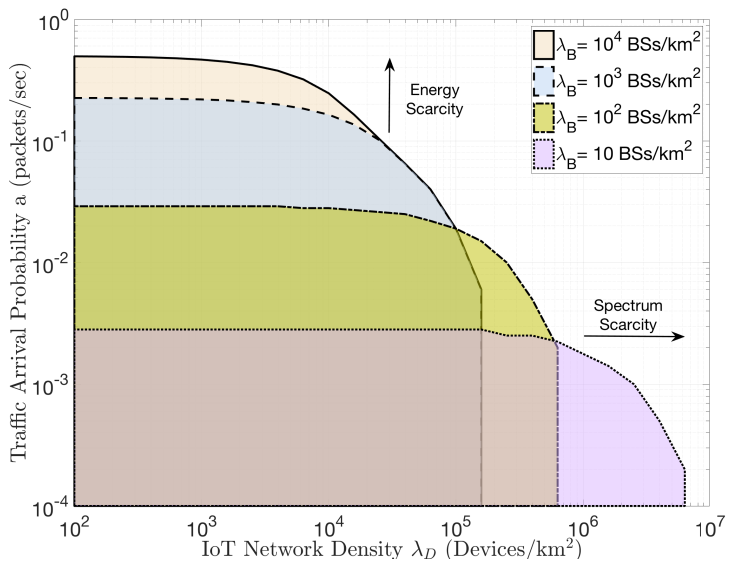

Figure 3. Stability Pareto regions $\left(a, \lambda_{D}\right)$ between the packet arrival probability $a$ in packets/sec versus the IoT network density $\lambda_{D}$ in IoTs $/ \mathrm{km}^{2}$ with different cellular network densities $\lambda_{B}$ in BSs $/ \mathrm{km}^{2}$, with $\theta=10 \mathrm{~dB}$.

unstable at $\theta \geq-5 \mathrm{~dB}$ and $\theta \geq 5 \mathrm{~dB}$, respectively. Note that for stable network operation, the transmission success probability is higher for the $a=0.1$ than that of the $a=0.2$. This is because more devices remain in the idle state (i.e., empty buffers) for the $a=0.1$ scenario, which reliefs interference and improves transmission success probability. For unstable network operation, the probability for being idle is zero irrespective of the actual value of $a$. Hence, the transmission success probabilities for $a=0.2$ and $a=0.1$ match in the unstable network regime.

Fig. 3 shows the sustainability regions versus the spatiotemporal IoT traffic density $\left(a, \lambda_{D}\right)$ for different intensities of cellular BSs. The shaded regions in Fig. 3 determine all pairs of $\left(a, \lambda_{D}\right)$ that lead to stable network operation. Hence, the harvesting rate is quick enough and the mutual interference is tolerable to maintain packet departure rates that are greater than the packet arrival rates. Hence, all generated packets get delivered to the intended receivers in finite time. Operating beyond that shaded regions means that the packet departure rates is less than the packet arrival rate, which leads to infinite packets accumulation in the IoT devices buffers and infinite average delay. Instability can occur due to i) energy scarcity (i.e., slow harvesting rates), ii) overwhelming interference (i.e., low transmission success probability), or both i) and ii).

Several insights to extend the sustainability region for IoT networks can be obtained from Fig. 3. For instance, the figure shows how to cope with higher traffic arrivals $a$ for low and medium device densities (i.e., up to $10^{4}$ device $/ \mathrm{km}^{2}$ ). In this region, energy scarcity is the prominent factor for instability. Hence, increasing the BSs intensity extend the sustainability region, for this range of device intensities, as it improves the harvesting rates to cope with the higher traffic arrivals. In contrast, at high devices density (beyond $10^{5}$ device $/ \mathrm{km}^{2}$ ), the interference becomes the dominant obstacle for stability, where several retransmissions are required to successfully deliver each packet. Hence, increasing the BS intensity do not extend the sustainability region for higher 
IoT device intensity. Interestingly, Fig. 3 shows that lower BS intensity can accommodate higher IoT devices intensity (e.g., for $a=0.001)$. Such counterintuitive behavior is because longer harvesting time defers IoT devices transmissions and suppresses the aggregate interference. Hence, energy harvesting acts as a distributed spectrum access coordination scheme, which improves transmission success probability and extends the sustainability region for higher IoT device density. This highlights the importance of distributed interference management for self-sustainable massive IoT networks.

\section{Conclusion}

Using stochastic geometry and queuing theory, this paper develops a spatiotemporal mathematical model for selfsustainable IoT networks that recycle the RF-energy of downlink cellular network. Particularly, a two-dimensional DTMC is used to track the time evolution of the data buffer and the battery of each IoT device. Then, IoT network is modeled as a network of spatially interacting DTMCs due to the mutual interference between IoT devices. To this end, joint probability of the number of packets in the buffers and the energy levels in the batteries is obtained. The developed model is then used to characterize the network parameters, in terms of spatiotemporal IoT traffic intensity and cellular network density, that lead to a self-sustainable IoT network. The results identify the scenarios where the IoT network fails to be self-sustainable due to energy scarcity, overwhelming interference, or both.

\section{REFERENCES}

[1] A. Al-Fuqaha, M. Guizani, M. Mohammadi, M. Aledhari, and M. Ayyash, "Internet of Things: A survey on enabling technologies, protocols, and applications," IEEE Communications Surveys Tutorials, vol. 17, no. 4, pp. 2347-2376, Fourthquarter 2015.

[2] A. Bader, H. ElSawy, M. Gharbieh, M. S. Alouini, A. Adinoyi, and F. Alshaalan, "First mile challenges for large-scale IoT," IEEE Communications Magazine, vol. 55, no. 3, pp. 138-144, March 2017.

[3] A. Laya, C. Kalalas, F. Vazquez-Gallego, L. Alonso, and J. AlonsoZarate, "Goodbye, ALOHA!" IEEE Access, vol. 4, pp. 2029-2044, 2016.

[4] Y. P. E. Wang, X. Lin, A. Adhikary, A. Grovlen, Y. Sui, Y. Blankenship, J. Bergman, and H. S. Razaghi, "A primer on 3GPP narrowband Internet of Things," IEEE Communications Magazine, vol. 55, no. 3, pp. 117-123, March 2017.

[5] R. S. Sinha, Y. Wei, and S.-H. Hwang, "A survey on LPWA technology: LoRa and NB-IoT," ICT Express, vol. 3, no. 1, pp. $14-21$, March 2017.

[6] X. Lu, P. Wang, D. Niyato, D. I. Kim, and Z. Han, "Wireless networks with RF energy harvesting: A contemporary survey," IEEE Communications Surveys Tutorials, vol. 17, no. 2, pp. 757-789, Secondquarter 2015.

[7] H. ElSawy, A. Sultan-Salem, M. S. Alouini, and M. Z. Win, "Modeling and analysis of cellular networks using stochastic geometry: A tutorial," IEEE Communications Surveys Tutorials, vol. 19, no. 1, pp. 167-203, Firstquarter 2017.

[8] N. Kouzayha, Z. Dawy, J. G. Andrews, and H. ElSawy, "Join downlink/uplink RF wake-up solution for IoT over cellular networks," IEEE Transactions on Wireless Communications, vol. 17, no. 3, pp. 1574-1588, March 2018.

[9] Z. Qin, Y. Liu, G. Y. Li, and J. A. McCann, "Modelling and analysis of low-power wide-area networks," in 2017 IEEE International Conference on Communications (ICC), May 2017, pp. 1-7.

[10] M. A. Kishk and H. S. Dhillon, "Joint uplink and downlink coverage analysis of cellular-based RF-powered IoT network," IEEE Transactions on Green Communications and Networking, vol. PP, no. 99, pp. $1-1,2017$.
[11] M. Gharbieh, H. ElSawy, A. Bader, and M. S. Alouini, "Spatiotemporal stochastic modeling of IoT enabled cellular networks: Scalability and stability analysis," IEEE Transactions on Communications, vol. 65, no. 8, pp. 3585-3600, August 2017.

[12] Y. Zhong, T. Q. S. Quek, and X. Ge, "Heterogeneous cellular networks with spatio-temporal traffic: Delay analysis and scheduling," IEEE Journal on Selected Areas in Communications, vol. 35, no. 6, pp. 1373-1386, June 2017.

[13] G. Chisci, H. ElSawy, A. Conti, M. S. Alouini, and M. Z. Win, "On the scalability of uncoordinated multiple access for the Internet of Things," in 2017 International Symposium on Wireless Communication Systems (ISWCS), August 2017, pp. 402-407.

[14] A. H. Sakr and E. Hossain, "Analysis of K-tier uplink cellular networks with ambient RF energy harvesting," IEEE J. Sel. Areas Commun., vol. 33, no. 10, pp. 2226-2238, October 2015.

[15] T. A. Khan, P. V. Orlik, K. J. Kim, R. W. Heath, and K. Sawa, "A stochastic geometry analysis of large-scale cooperative wireless networks powered by energy harvesting," IEEE Transactions on Communications, vol. 65, no. 8, pp. 3343-3358, August 2017.

[16] I. Flint, X. Lu, N. Privault, D. Niyato, and P. Wang, "Performance analysis of ambient RF energy harvesting: A stochastic geometry approach," in 2014 IEEE Global Communications Conference, December 2014, pp. 1448-1453.

[17] H. S. Dhillon, Y. Li, P. Nuggehalli, Z. Pi, and J. G. Andrews, "Fundamentals of base station availability in cellular networks with energy harvesting," in 2013 IEEE Global Communications Conference (GLOBECOM), December 2013, pp. 4110-4115.

[18] M. Haenggi, "The meta distribution of the SIR in Poisson bipolar and cellular networks," IEEE Transactions on Wireless Communications, vol. 15, no. 4, pp. 2577-2589, April 2016.

[19] Z. Gong and M. Haenggi, "Interference and outage in mobile random networks: Expectation, distribution, and correlation," IEEE Transactions on Mobile Computing, vol. 13, no. 2, pp. 337-349, February 2014.

[20] A. S. Alfa, Applied Discrete-Time Queues, 2nd ed. Springer Publishing Company, Incorporated, 2015.

[21] I. Krikidis, T. Charalambous, and J. S. Thompson, "Buffer-aided relay selection for cooperative diversity systems without delay constraints," IEEE Transactions on Wireless Communications, vol. 11, no. 5, pp. 1957-1967, May 2012.

[22] Y. Zhou and W. Zhuang, "Performance analysis of cooperative communication in decentralized wireless networks with unsaturated traffic," IEEE Trans. Wireless Commun., vol. 15, no. 5, pp. 35183530, May 2016.

[23] I. S. Gradshteyn and I. M. Ryzhik, Table of Integrals, Series, and Products, 5th ed. San Diego, CA: Academic, 1994.

\section{APPENDIX A}

Laplace Transform of InTERfering IoT Devices

Due to the independence between the channels and the moment generating function (MGF) of the exponential distribution, the LT of the aggregate interference in the IoT network is given by

$$
\mathcal{L}_{D}(s)=\mathbb{E}_{y_{j}}\left[\prod_{y_{j} \in \tilde{\Phi}_{D} \backslash y_{0}} \frac{1}{1+s P_{D}\left\|y_{j}\right\|^{-\alpha_{D}}}\right]=\exp \left(-\frac{2 \pi^{2} d \lambda_{D} P_{D}^{\frac{2}{\alpha_{D}}}}{\alpha_{D} \sin \left(\frac{2 \pi}{\alpha_{D}}\right)} s^{\frac{2}{\alpha_{D}}}\right),
$$

which is obtained using the probability generating function (PGFL) of $\Phi_{D} \backslash y_{0}$, and using [23, (4,3.241.2)].

\section{APPENDIX B}

\section{Statistics of Harvested Power}

First, we derive the LT of the aggregate downlink power in a similar way as in Appendix A as $\mathcal{L}_{C}(s)=\exp \left(-\kappa_{0} s^{\frac{2}{\alpha_{B}}}\right)$. Then, we invert $\mathcal{L}_{C}(s)$ via applying the Bromwich inversion theorem to show that the CDF of $P_{H}$ is given by (5). For $\alpha_{B}=4$, the CDF of $P_{H}$ is deduced using [23, 3.953.6]. 